\title{
Dokumente
}

\section{Ausführungen Chruschtschows auf der Sitzung der Regierungsdelegationen von UdSSR und DDR am 19. September 1955}

Die sowjetische Regierung ließ den Verhandlungen mit Adenauer vom 9. bis 13. September 1955 über die Aufnahme diplomatischer Beziehungen zur Bundesrepublik Deutschland drei Tage später Beratungen mit den Vertretern der DDR über die Gestaltung des beiderseitigen Verhältnisses folgen, in denen es um die Souveränität des ostdeutschen Staates und die der Sowjetunion weiter vorbehaltenen Rechte ging. Nach dem Fazit, das Nikolaj Bulganin als Vorsitzender des Ministerrates der UdSSR zog, äußerte sich die Chef der KPdSU wie folgt:

Chruschtschow: Ich möchte sagen, dass nach unserer Ansicht die enge Zusammenarbeit zwischen den politischen Parteien, die in der Nationalen Front des demokratischen Deutschlands vereint sind, eine große Bedeutung für die Entwicklung der Deutschen Demokratischen Republik hat.

Wir alle verstehen sehr gut, dass die Zusammenarbeit dieser Parteien und ihrer Führungen im Kampf um die Erzielung großer Erfolge bei der Entwicklung der Wirtschaft und Kultur der Deutschen Demokratischen Republik, bei der Herstellung freundschaftlicher Beziehungen zwischen der Deutschen Demokratischen Republik und der Sowjetunion sowie der weiteren Festigung dieser Beziehungen eine große Rolle spielt.

Hinsichtlich der Bedeutung der Nationalen Front möchte ich auch das nützliche Wirken des Vorsitzenden des Nationalrats der Nationalen Front des demokratischen Deutschlands, Professor [Erich] Correns, erwähnen.

Die aufrichtige und allseitige Zusammenarbeit zwischen den Parteien der Nationalen Front ist das Unterpfand für euer weiteres Voranschreiten. Deswegen möchte ich den Wunsch nach weiteren Erfolgen in der Entwicklung einer engen Zusammenarbeit zwischen den Parteien mit dem Ziel des weiteren Aufschwunges der Wirtschaft der DDR und des Wohlstandes ihrer Bevölkerung, zum Wohle des deutschen Volkes und des allgemeinen Friedens, zum Ausdruck bringen.

Uns ist klar, diese Zusammenarbeit zu erzielen - das ist keine einfache Aufgabe. Im Leben ist es nicht leicht, ein wechselseitiges Verstehen und Zusammenwirken in allen Fragen zu realisieren. Schließlich entstehen auch Fragen, bei denen verschiedene Standpunkte aufeinander treffen. Bei der Regelung solcher Fragen ist es erforderlich, politische Weisheit zu zeigen. Bei einer Parteienkoalition muss man die Interessen der Parteien einkalkulieren, die dazu gehören und Wege zur Regelung aller Fragen suchen und finden. Dabei muss man von der Notwendigkeit gegenseitiger Zugeständnisse ausgehen, doch diese Zugeständnisse dürfen nicht dem grundlegenden Ziel schaden, das sich die Deutsche Demokratische Republik stellt. Nur so kann man die Koalition der Parteien festigen und folglich die erzielten Errungenschaften der Deutschen Demokratischen Republik vermehren. 
Die Aufgabe, vor der das deutsche Volk steht und der die Aufmerksamkeit von Millionen Deutschen gilt, ist die Wiedervereinigung des deutschen Volkes in einem einheitlichen deutschen friedliebenden demokratischen Staat. Wir sind jederzeit bereit, mit allen Kräften zur erfolgreichen Lösung dieser Aufgabe beizutragen. Wir sind Realisten; bei der Regelung aller politischen Fragen und bei der Regelung dieser konkreten Frage müssen wir die Gegebenheiten nüchtern beurteilen. Die Wiedervereinigung des deutschen Staates in einer Weise, die sowohl dem deutschen Volk als auch den Völkern Europas nützt, hängt nicht nur von einer Seite ab. Hier gibt es zwei Seiten: die Deutsche Demokratische Republik und die Bundesrepublik Deutschland. Außerdem gibt es noch vier Staaten, die durch bestimmte Bedingungen und Verpflichtungen [miteinander] verbunden sind, und deren Ansichten zur Lösung der deutschen Frage bis jetzt noch auseinander gehen. Deswegen dürfen wir keine falschen Illusionen wecken.

Bekanntlich steht in Kürze das Treffen der Außenminister der vier Mächte in Genf an, wo es unter anderem auch um die Deutschland-Frage geht. Unser Standpunkt in dieser Hinsicht ist klar. In meinen Ausführungen möchte ich jetzt auf konkrete Fakten eingehen. Die Fakten sind folgende: Von den vier Mächten, deren Außenminister in Genf zusammenkommen, sind drei gehören dem Nordatlantischen Block (der NATO) an und einer nicht. Mehr noch: Die NATO ist gegen diesen Staat gerichtet. Die Regierungen der drei Staaten wollen zusammen mit der Regierung der Deutschen Bundesrepublik, dass die Wiedervereinigung Deutschlands zu ihren Bedingungen erfolgt und dass das wiedervereinigte Deutschland ebenfalls der NATO beitritt. Ich denke, dass ein solcher Weg nicht den Interessen des deutschen Volkes entspricht, dass er nicht den Interessen der Sicherheit der Völker Europas und der Verminderung der internationalen Entspannung entspricht. Wir können in keiner Weise einem solchen Gang der Geschehnisse zustimmen, in dessen Ergebnis der gegen die Sowjetunion und die Länder der Volksdemokratien gerichtete nordatlantische Militärblock (die NATO) gestärkt werden würde. Daher haben wir, als die Verhandlungen mit den Vertretern der Deutschen Bundesrepublik ${ }^{4}$ stattgefunden haben, Herrn Adenauer in aller Offenheit gesagt: Fordern Sie von uns nicht, was wir nicht geben können. Wir können Ihren Plan zur Wiedervereinigung Deutschlands nicht unterstützen wir können die NATO nicht unterstützen - eine Organisation, die gegen uns, gegen die Sache des Friedens, gerichtet ist. Ich denke, dass die Deutschen im entsprechenden Fall ebenso vorgehen würden.

Die Unterzeichnung und das Inkrafttreten der Pariser Verträge, der Beitritt der Deutschen Bundesrepublik zur NATO5 haben ernste Hindernisse geschaffen für die Wiedervereinigung der zwei Teile Deutschlands zu einem einheitlichen deutschen Staat.

Es stellt sich die Frage: Wie werden die Hoffnungen des deutschen Volkes auf Wiedervereinigung Befriedigung finden; soll denn die bestehende Lage immer so

4 Chruščëv vermied den amtlichen Staatsnamen Bundesrepublik Deutschland, um deutlich zu machen, dass es sich nur um einen Teil handele, der nicht für das Ganze sprechen könne.

5 Aufgrund der Pariser Verträge vom 23. Oktober 1954 wurde die Bundesrepublik Deutschland am 5. Mai 1955 in die NATO aufgenommen. 
bleiben? Darauf antworten wir klar und bestimmt: Die Deutschen sollen diese Frage selbst entscheiden. Deutsche, [setzt euch] an einen Tisch! Die deutsche Frage kann keiner besser lösen als die Deutschen selbst. Die Wiedervereinigung Deutschlands muss so erfolgen, dass der einheitliche deutsche Staat friedliebend ist, demokratisch, dass die Kräfte des deutschen Staates nicht gegen andere Staaten gerichtet werden. Eine solche Regelung der deutschen Frage werden wir auf jede Weise unterstützen.

Es ist klar, dass unter den gegenwärtigen Bedingungen die Regelung der deutschen Frage eine schwierige Sache ist. Wir haben kürzlich Zusammenkünfte und Gespräche mit Vertretern der Deutschen Bundesrepublik gehabt. Die Verhandlungen mit den Vertretern der BRD haben gezeigt, dass es auf dem Weg zur raschen Regelung dieser Frage große Schwierigkeiten gibt. Als die Verhandlungen mit der Delegation der Deutschen Bundesrepublik stattfanden, hat mir Herr Adenauer in einer Pause einzureden versucht, dass der Nordatlantische Block für friedliche Ziele geschaffen worden sei, so als würde diese Organisation in keiner Weise die Sowjetunion bedrohen.

Doch wir wissen genau, dass die Pariser Verträge militärische Verträge sind. Wir wissen auch, dass die NATO als militärische Organisation geschaffen wurde, ${ }^{6}$ dass die NATO einen Oberbefehlshaber ihrer Streitkräfte in Europa hat: General Gruenther. Folglich ist die NATO keine Sportorganisation, sie wurde nicht zur Vorbereitung auf sportliche Wettkämpfe geschaffen, auch nicht für Fußballspiele.

Bekanntermaßen wird Gruenther nicht als Trainer einer Fußballmannschaft aufgeführt; alle kennen ihn als General, der „die Mannschaft“ für den Krieg „trainiert“ und zwar für den Krieg gegen die Sowjetunion und die Länder der Volksdemokratie. Das ist nicht zu übersehen.

Man hat versucht uns zu beweisen, dass die NATO zu Zwecken der Verteidigung geschaffen wurde. Wir haben uns seinerzeit an die Mitglieder der NATO gewandt und gesagt: Wenn die NATO zur Verteidigung geschaffen wurde, möchten wir dieser „Verteidigungsorganisation“ ebenfalls beitreten.7 Doch be-

6 Das trifft insofern nicht ganz zu, als bei Abschluss des Nordatlantikvertrags am 4. April 1949 noch nicht vom Aufbau militärischer Strukturen die Rede war. Es handelte sich zunächst nur um einen politischen Vertrag, der die USA zum Eintreten für die Verbündeten in Europa verpflichtete. Da man davon ausging, dass die UdSSR sich dadurch von einem Angriff abgehalten sehen würde, schien die Sicherheit der Bundesgenossen auf diese Weise gewährleistet. Erst nachdem der Angriff Nordkoreas am 25. Juni 1950 im Westen die Sorge hervorgerufen hatte, dass die Kommunisten auch in Europa zu bewaffneten Aktionen übergehen könnten, begann ab Anfang 1951 das atlantische Bündnis den Aufbau einer militärischen Organisation.

7 Am 31. März 1954 richtete die UdSSR eine Note an die drei Westmächte, in der sie zunächst ihren auf der Berliner Konferenz am 10. Februar 1954 vorgelegten Vorschlag eines gesamteuropäischen Systems der kollektiven Sicherheit wiederholte und der westlichen These widersprach, das atlantische Bündnis habe eine defensive Ausrichtung. Abschließend hieß es, dass die Organisation des Nordatlantikpakts „unter entsprechenden Bedingungen ihren aggressiven Charakter verlieren könnte, wenn alle Großmächte, die zur Anti-Hitler-Koalition gehörten, an ihr teilnehmen würden“. Dieser Vorschlag zielte von vornherein auf Ablehnung, denn es war klar, dass eine derart veränderte Allianz nicht mehr dem Zweck dienen würde, ihre Mitglieder vor der Sowjetunion zu schützen. Damit sollte der Öffentlichkeit der „aggressive Charakter“ der NATO vor Augen geführt werden. 
kanntlich wurde die Sowjetunion nicht aufgenommen. Auch diese Tatsache zeigt, dass die NATO gegen die Sowjetunion gerichtet ist.

Es ist völlig ofensichtlich, dass das Inkrafttreten der Pariser Verträge und der Beitritt der BRD zur NATO ein ernstes Hindernis für die Regelung der deutschen Frage geschaffen haben. Wir schlagen den einzig richtigen Ausweg vor: die Regelung dieser Frage in die Hände des deutschen Volkes zu legen. ${ }^{8}$ Die Deutschen werden Wege finden, die zur richtigen Lösung des Problems führen.

Ich denke, ich bringe unsere gemeinsame Ansicht zum Ausdruck, wenn ich sage, dass die deutsche Nation und die Völker der Sowjetunion daran interessiert sind friedliche, freundschaftliche Beziehungen [miteinander] zu haben.

Ich will sagen, dass offenbar für eine gewisse Zeit damit zu rechnen ist, dass in Deutschland zwei Staaten bestehen: die Deutsche Demokratische Republik und die Deutsche Bundesrepublik.

Zwischen der Sowjetunion und der Deutschen Demokratischen Republik sind aufrichtig freundschaftliche Beziehungen entstanden. Die sowjetische Regierung wird sich ebenfalls auf jede Weise um die Anbahnung freundschaftlicher Beziehungen zur Deutschen Bundesrepublik bemühen und danach streben, eine Annäherung der beiden Teile Deutschlands zu fördern.

Ich möchte als Kommunist und als Sekretär des Zentralkomitees der Kommunistischen Partei der Sowjetunion sagen, ohne allen Anwesenden meinen Standpunkt aufzunötigen, dass unsere Partei, die sich von der Lehre von Marx, Engels, Lenin und Stalin leiten lässt, sich das edelste Ziel setzt: den Aufbau des Kommunismus. ${ }^{9}$ Wir streben danach, dass es keine Ausbeutung des Menschen durch den Menschen [mehr] gibt, dass der Nutzen der Arbeit und der Wissenschaft dem ganzen Volke dient. Wenn sich alle Völker auf einen solchen Entwicklungsweg begäben, würde dies nicht nur die Möglichkeit von Kriegen, sondern auch jedweder bewaffneter Konflikte ausschließen.

Die Werktätigen der Deutschen Demokratischen Republik haben den Weg des sozialistischen Aufbaus beschritten. Das ist ein großes historisches Ereignis im Leben des deutschen Volkes. Und auf diesem Wege habt ihr unsere Unterstützung. Wir wünschen euch vollen Erfolg bei eurer großen schöpferischen Tätigkeit. Wir sind davon überzeugt, dass ihr neue Siege auf diesem Weg zu erringen imstande seid. Der Aufbau des Sozialismus in der Deutschen Demokratischen Republik ist eine so wichtige Aufgabe, dass es jetzt sogar schwer fällt, die richtigen Worte zu finden, um die ganze Bedeutung und Größe dieser Sache zum Ausdruck zu bringen.

Der Aufbau des Sozialismus in der Deutschen Demokratischen Republik entspricht den Interessen aller Werktätigen Deutschlands. Viele Arbeiter in den Reihen der deutschen sozialdemokratischen Partei, der deutschen christlich-demokratischen Partei und anderen Parteien werden sich bemühen, beim Aufbau des

8 Die sowjetische Führung vertrat den Standpunkt, die „Deutschen selbst“, das heißt ihre politischen Vertreter, hätten die Frage ihrer staatlichen Einheit zu entscheiden, seit der Pariser VierMächte-Konferenz im Sommer 1949. Damit war festgelegt, dass nur eine Regelung in Betracht kam, der das SED-Regime zustimmen würde.

9 Nach der amtlichen sowjetischen Terminologie war der Kommunismus das höchste Stadium des Sozialismus, in dem man das ideale Endziel der gesellschaftlichen Entwicklung erreichen werde. 
sozialistischen deutschen Staates mitzuwirken. Doch der Kampf für den Sozialismus, für den Aufbau des sozialistischen deutschen Staates - das ist natürlich eine innere Angelegenheit der deutschen Arbeiterklasse, des deutschen Volkes.

Ich muss sagen, dass die Aufgabe des sowjetischen Staates lautet, gute gutnachbarliche, freundschaftliche Beziehungen zum ganzen deutschen Volk herzustellen. Wir zweifeln nicht daran, dass sich die Führung der Deutschen Demokratischen Republik ebenfalls darum bemüht.

Der Kanzler der Bundesrepublik Deutschland, Herr Adenauer, hat in den Verhandlungen in Moskau ebenfalls erklärt, dass die Regierung der Deutschen Bundesrepublik freundschaftliche Beziehungen zum sowjetischen Volk zu entwickeln sucht. Ich habe kein Recht, diese Erklärung anzuzweifeln. Wir werden eine Bekräftigung dieser guten Worte mit guten Taten begrüßen.

Wir wissen, dass bei den Vertretern der Deutschen Demokratischen Republik, mit der wir erfolgreiche Verhandlungen führen, ihre Worte und Zusagen nicht von der praktischen Tätigkeit abweichen, und wir sind davon überzeugt, dass dies auch künftig so sein wird. Unsere Freundschaft und das gegenseitige Vertrauen werden mit jedem Tag stärker.

Wir hoffen ebenfalls, dass sich im weiteren Verlauf die Beziehungen zwischen der Sowjetunion und der Deutschen Bundesrepublik zufriedenstellend entwickeln. Doch wie es im russischem Sprichwort heißt: Die Zeit wird es zeigen. Wir von unserer Seite werden alles tun, damit sich die Beziehungen zwischen der Sowjetunion und der Deutschen Bundesrepublik erfolgreich entwickeln. Sowohl die Sowjetunion als auch die Deutsche Bundesrepublik sind daran interessiert, dass sich zwischen unseren Ländern die Handels- und Kulturbeziehungen auf breiter Front entwickeln. Das wird der Deutschen Bundesrepublik, der Deutschen Demokratischen Republik und die Sowjetunion großen Vorteil bringen.

Ich will mich ebenfalls zu einer privaten Frage äußern: zu den früheren Kriegsgefangenen, die von einem Gericht der Sowjetunion als Kriegsverbrecher verurteilt wurden. Sie erinnern sich, dass über diese Frage Gen. Bulganin und ich einen Meinungsaustausch mit Ihnen hatten, als wir auf dem Rückweg aus Genf in Berlin Station machten. ${ }^{10}$ In dieser Frage hatte sich der Präsident der Deutschen Demokratischen Republik, Gen. Pieck, mit einem Brief an den Vorsitzenden des Präsidiums des Obersten Sowjets, Gen. Woroschilow, gewandt.

Als die Delegation der Deutschen Bundesrepublik zu uns kam, stellte sie uns ebenfalls diese Frage und stellte sie in einen Zusammenhang mit der Aufnahme der diplomatischen Beziehungen zwischen der UdSSR und der DBR. Wir haben Herrn Adenauer und den Mitgliedern der Regierungsdelegation der Deutschen Bundesrepublik erklärt, dass diese Frage keinerlei Bezug aufweist zu den Verhandlungen über die Aufnahme der diplomatischen Beziehungen und dass sie nur die Sowjetunion entscheiden kann, weil diese Leute Verbrechen am sowjetischen Volk begangen haben und dafür von einem sowjetischen Gericht verurteilt worden sind. Doch die Zeit geht vorbei, und Menschen ändern sich. Die einen

10 Nach der Genfer Gipfelkonferenz unterbrachen Chruščëv und Bulganin die Reise nach Moskau vom 24. bis 27. Juli 1955, um der SED-Führung zu versichern, dass sich an der sowjetischen Haltung in der deutschen Frage nichts geändert habe.eimreis 
ändern sich zum Besseren, die anderen zum Schlechteren. Doch die Mehrzahl der Leute ändert sich in besserem Sinne. Daher sind wir der Ansicht, dass man der Bitte um vorzeitige Entlassung dieser Leute entsprechen kann. Wir berücksichtigen dabei, dass sie Verwandte und Angehörige haben, die auf sie als Väter, Söhne, Ehepartner und Brüder warten. Wir verstehen, dass man auf diese menschlichen Gefühle Rücksicht nehmen muss. Diese Leute haben schon eine erhebliche Zeit ihrer Gefangenschaft verbüßt, und wir hoffen, dass sie den Großmut der sowjetischen Regierung zu schätzen wissen und sich nach ihrer Entlassung gegenüber der Sowjetunion nicht vom Gefühl der Rache leiten lassen.

Das sind die Fragen, die ich in meiner Stellungnahme ansprechen wollte.

Der Vertragsentwurf, den die [Verhandlungs-]Kommission vorbereitet hat, ${ }^{11}$ stellt offenbar beide Seiten zufrieden, wie das nicht so oft der Fall ist. Meistens ist es so, dass man die Regelung durch Kompromiss findet. Wir haben keine Kompromisse, weil es keinen Wettstreit zwischen unterschiedlichen Standpunkten gibt, sondern eine Gemeinsamkeit der Ansichten, eine Gemeinsamkeit der Weltanschauung. Zwar mag die Tatsache, dass unsere sowjetischen Streitkräfte vorerst auf eurem Hoheitsgebiet verbleiben, einen gewissen Verdruss hervorrufen, aber wir sind sicher, dass die Notwendigkeit dieser Maßnahme richtig verstanden werden wird. Wir sind uns darüber klar, dass die Anwesenheit ausländischer Streitkräfte selbst einer befreundeten Macht oft keine Begeisterung bei der Bevölkerung hervorruft. Trotzdem können und sollen die Leute die Notwendigkeit angesichts der vorliegenden Bedingungen einsehen. Unsere gemeinsame Entscheidung über den Verbleib der sowjetischen Streitkräfte auf euerem Territorium ist eine zeitweilige Maßnahme. Wir sind uns bewusst, dass dies eine unangenehme Sache ist, und wenn hier jemand sagen würde, dass das eine angenehme Entscheidung ist, würden wir ihm nicht trauen. Sind Sie damit einverstanden?

Ulbricht: Einverstanden.

Chruschtschow: Aber wir werden gemeinsam mit Ihnen beharrlich auf eine Situation hinarbeiten, dass zwischen den Staaten eine Vereinbarung erzielt wird über die Reduzierung der Streitkräfte insgesamt und über den Abzug der sowjetischen, amerikanischen, englischen und französischen Streitkräfte, die sich in Deutschland befinden. Wenn die USA, England und Frankreich schon heute

11 Die Verhandlungen wurden am 20. September 1956 abgeschlossen mit einem „Vertrag über die Beziehungen zwischen der Sowjetunion und der DDR“. Darin hieß es, die Beziehungen zwischen beiden Ländern beruhten „auf völliger Gleichheit, gegenseitiger Achtung der Souveränität und der Nichteinmischung in die inneren Angelegenheiten“. Die DDR sei „frei in der Entscheidung über die Fragen ihrer Innenpolitik und Außenpolitik einschließlich der Beziehungen zur Deutschen Bundesrepublik“. Mit Zustimmung ihrer Regierung sollten die auf ihrem Territorium stationierten sowjetischen Truppen dort „zeitweilig“ verbleiben. In einem Briefwechsel zwischen DDR-Außenminister Lothar Bolz und Valerian Zorin als stellvertretendem Außenminister der UdSSR wurde festgelegt, dass die DDR die „Bewachung und Kontrolle an den Grenzen der DDR, an der Demarkationslinie zwischen der DDR und der Deutschen Bundesrepublik, am Außenring von Groß-Berlin sowie auf den im Gebiet der DDR liegenden Verbindungswegen zwischen der Deutschen Bundesrepublik und West-Berlin ausübt“. Zugleich blieb die Kontrolle des Verkehrs von Militärpersonal und Gütern der in West-Berlin stationierten Garnisonen der USA, Großbritanniens und Frankreichs zwischen der Bundesrepublik und West-Berlin den Streitkräften der UdSSR vorbehalten. 
dazu bereit wären, könnten wir diese Frage jetzt auf der Stelle regeln. Wir sind bereit, unsere Streitkräfte aus Deutschland abzuziehen, freilich unter der Bedingung, dass unsere Partner im Krieg gegen Hitlerdeutschland ihre Truppen abziehen.

Wir haben die Hoffnung auf Regelung dieser Frage in der Zukunft. Die Genfer Konferenz der Regierungschefs der vier Mächte hat diese Hoffnung geweckt. Wir hoffen, dass sich der Geist von Genf entwickeln und festigen wird, aber nicht als unsichtbarer und nicht wahrzunehmender Geist, sondern in Form konkreter Taten, wie dies von unserer Seite der Fall gewesen ist, als wir unsere Streitkräfte um 640000 Mann reduziert haben, 12 als wir auf die Verpachtung des Marinestützpunkts Porkkala-Udd in Finnland verzichtet haben ${ }^{13}$ und eine Reihe weiterer Maßnahmen durchgeführt haben. Wir warten darauf, dass unsere Partner in Genf in gleicher Weise reagieren, woraufhin wir unsererseits neue Vorschläge unterbreiten könnten.

Manche bürgerlichen Politiker warfen uns vor, dass wir zwar lächelten und uns freundschaftlich zeigten, jedoch angeblich nicht darüber hinaus gegangen sind. Sind denn die von mir angeführten Tatsachen der Streitkräftereduzierung und des Verzichts - sind das denn etwa keine konkreten Taten, die auf eine Abmilderung der internationalen Spannungen abzielen?

Gleichzeitig warten wir auf die konkreten Ergebnisse des Geistes von Genf aufseiten der Westmächte, die zur Abmilderung der internationalen Spannungen und zur Festigung der Sache des Friedens beitragen würden.

Übersetzt aus dem russischen Originaltext

RGANI, fond 52, opis' 1 , delo 557, Bl. 1-10

\section{Gespräch Chruschtschows mit dem Vorsitzenden der Sozialistischen Partei Italiens, Pietro Nenni, am 15. Oktober 1955}

Nach kurzen Ausführungen Chruschtschows über die UdSSR und die anderen sozialistischen Staaten verlief das Gespräch mit Pietro Nenni ${ }^{14}$ nach Aussage des sowjetischen Protokolls wie folgt.

Genosse Chruschtschow beleuchtete ausführlich die wichtigsten Maßnahmen der Sowjetunion zur Entspannung der internationalen Lage ${ }^{15}$ und ihre grundlegende Bedeutung für die Festigung der Position der Verfechter des Friedens in der gan-

12 Nach offizieller Erklärung wurden das Personal der sowjetischen Streitkräfte 1955 in zwei Schritten um insgesamt 640000 Mann reduziert.

13 Bei der Erneuerung des Beistandsvertrages mit Finnland am 19. September 1955 erklärte sich die UdSSR zur Räumung dieses Stützpunktes bereit, der im Friedensvertrag von 1947 für 50 Jahre an sie verpachtet worden war.

14 Nennis Sozialistische Partei stand - anders als die schwächeren, westlich orientierten Sozialdemokraten von Giuseppe Saragat - auf der Seite der UdSSR.

15 Chruščëv hatte im Kreml gegen den Widerstand von Außenminister Vjačeslav Molotov den Abschluss des Staatsvertrags mit Österreich am 15. Mai 1955 durchgesetzt, der das Ende der Besetzung durch die Vier Mächte und die Unabhängigkeit des Landes auf der Grundlage der von diesem erklärten Neutralität festlegte. Vom 26. Mai bis 2. Juni 1955 war er zusammen mit dem Vor- 